\title{
Influence of a diet and physical activity on the nutritional status of soldiers of the Polish Air Cavalry units
}

\author{
Jerzy Bertrandt ${ }^{1}$, Anna Anyzewska ${ }^{1}$, Roman Lakomy $^{1}$, Tomasz Lepionka ${ }^{1}$, Ewa Szarska ${ }^{2}$, \\ Ewelina Maculewicz ${ }^{2}$ and Andrzej Tomczak ${ }^{3}$ \\ ${ }^{1}$ Laboratory of Food and Nutrition Hygiene, Military Institute of Hygiene and Epidemiology, Warsaw, Poland, \\ ${ }^{2}$ Laboratory of Physiology, Military Institute of Hygiene and Epidemiology, Warsaw, Poland and \\ ${ }^{3}$ The War Studies University in Warsaw, Faculty of National Security, Department of Security Education, Warsaw, \\ Poland
}

\begin{abstract}
Introduction: Research from recent years indicate a problem of excessive body weight among soldiers, who due to the kind of carried out work should reveal the best health and fitness. Moreover, proper nutritional status in case of soldiers is extremely important, as it significantly affects their physical fitness. The aim of the study was to determine impact of the diet and physical activity on selected indicators of protein-energy and mineral nutritional status.
\end{abstract}

Materials and methods: One hundred and twenty male soldiers (aged: $28 \pm 5$; years of service: $5 \pm 5$ ), serving completed validated for Polish population the Food Frequency Questionnaire and long-form International Physical Activity Questionnaire. Body composition was determined by an electric bioimpedance method (TANITA MC-780) and bone mineral density of forearm bone of the nondominant hand was assessed by the DEXA densitometric method, using apparatus EXA 3000.

Results and discussion: This study confirmed the impact of both the diet and physical activity on selected measures of soldiers' nutritional status, i.e. fat mass index and bone mineral density. Out of selected 61 products negative correlation between FMI and frequency of apples and pears consumption $(r=-0.23, p=0,023)$, and positive correlation with frequency of beer $(r=0.20, p=0.049)$ and vodka and spirits drinking $(r=0.26, p=0.010)$ consumption were found. While the T-score value positively correlated with frequency of tropical fruits $(r=0.23, p=0.024)$, avocado $(r=0.25, p=0.014)$, fine-grained not refined groats $(r=0.22, p=0.030)$ and eggs $(r=0.29, p=0.004)$ consumption. A negative correlation was found for frequency of refined bread $(r=-0.24, p=0.019)$, sausages $(r=-0.35, p<0.023)$, high-quality cold cuts $(r=-0.25, p=0.014$, sugar to sweeten beverages $(r=-0.31, p=0.002)$, biscuits and cakes $(r=-0.23, p=0.016)$, salty snacks $(r=-0.23, p=0.023)$, fruit juices and nectars $(r=-0.28, p=0.005)$ and sugar-sweetened beverages $(\mathrm{r}=-0.34, \mathrm{p}=0.001)$. A negative correlation with FMI $(\mathrm{r}=-0.22, \mathrm{p}=0.036)$ for weekly physical activity expressed in MET-minutes/week was found. Positive correlations were found between T-score value and physical activity expressed in MET-minutes per week in total, housework, house maintenance and caring for family $(\mathrm{r}=0.22 ; \mathrm{p}=0.040)$, and recreation, sport and physical activity in leisure time $(r=0.25, p=0.019)$, as well as moderate physical activity $(r=0.28, p=0.008)$ and intense one $(\mathrm{r}=0.21, \mathrm{p}=0.046)$. The founded abnormalities in nutritional status, as well as numerous nutritional errors indicate a need for further monitoring of this group of soldiers, not only in terms of accuracy of nutritional status, their diet and physical activity, but also wider understood health behavior

\section{Conflict of Interest}

There is no conflict of interest 